\title{
ON A TWO-POINT BOUNDARY VALUE PROBLEM FOR THIRD-ORDER LINEAR FUNCTIONAL DIFFERENTIAL EQUATIONS. PART I.
}

\author{
ROBERT HAKL
}

Abstract. Efficient conditions sufficient for the solvability of the problem

$$
u^{\prime \prime \prime}(t)=\ell(u)(t)+q(t) ; \quad u(a)=c_{1}, \quad u^{\prime}(a)=c_{2}, \quad u(b)=c_{3}
$$

are established. Here $\ell: C([a, b] ; \mathbb{R}) \rightarrow L([a, b] ; \mathbb{R})$ is a linear bounded operator, $q \in L([a, b] ; \mathbb{R})$ and $c_{i} \in \mathbb{R}(i=1,2,3)$. Sign-constant solutions are discussed as well.

\section{INTRODUCTION}

Theory of boundary value problems for functional differential equations and systems has been recently made more complete due to an effort of many mathematicians. For an overview of the results known nowadays one is advised to see monographs $[1,2,4-6,9,11-16,18]$ and references therein.

In the presented paper, we will consider the two-point boundary value problem

$$
\begin{aligned}
& u^{\prime \prime \prime}(t)=\ell(u)(t)+q(t) \quad \text { for a.e. } t \in[a, b], \\
& u(a)=c_{1}, \quad u^{\prime}(a)=c_{2}, \quad u(b)=c_{3},
\end{aligned}
$$

where $\ell: C([a, b] ; \mathbb{R}) \rightarrow L([a, b] ; \mathbb{R})$ is a linear bounded operator, $q \in L([a, b] ; \mathbb{R})$, $c_{i} \in \mathbb{R}(i=1,2,3)$. By a solution to the problem (1.1), (1.2) we understand a function $u:[a, b] \rightarrow \mathbb{R}$ which is absolutely continuous together with its first and second derivatives, satisfies the equality (1.1) almost everywhere in $[a, b]$ and (1.2) holds. Efficient conditions guaranteeing the unique solvability of the problem (1.1), (1.2) are established in the paper. A great importance is put on the question, whether there exist solutions which are positive in the interval $] a, b[$. As far as the author is aware there is no article dealing exactly with the problem (1.1), (1.2).

This paper is the first part of the research dealing with the equation (1.1), when the operator on the right-hand side of the equation has a general form. The second part, dealing with the particular case when the equation (1.1) is an equation with deviating arguments, is a subject of the forthcoming paper. The presented paper is split into three sections - the first one is an introduction, the second one contains the list of the results obtained and the third one is devoted to their proofs.

$M S C$ (2010): primary 34K06, 34K10.

Keywords: Linear functional differential equation, boundary value problem, positive solution.

The research was supported by RVO: 67985840 . 
The following notation is used throughout the paper:

$\mathbb{R}$ is a set of all real numbers, $\mathbb{R}^{+}=[0,+\infty[$.

$C([a, b] ; \mathbb{R})$ is a Banach space of all continuous functions $u:[a, b] \rightarrow \mathbb{R}$ endowed with the norm

$$
\|u\|_{C}=\max \{|u(t)|: t \in[a, b]\} .
$$

$C\left([a, b] ; \mathbb{R}^{+}\right)=\left\{u \in C([a, b] ; \mathbb{R}): u(t) \in \mathbb{R}^{+}\right.$for $\left.t \in[a, b]\right\}$.

$\widetilde{C}^{2}([a, b] ; \mathbb{R})$ is a set of all functions $u:[a, b] \rightarrow \mathbb{R}$ which are absolutely continuous together with their first and second derivatives.

$\widetilde{C}_{l o c}^{2}(] a, b[; \mathbb{R})$ is a set of all functions $\left.u:\right] a, b\left[\rightarrow \mathbb{R}\right.$ such that $u \in \widetilde{C}^{2}([\alpha, \beta] ; \mathbb{R})$ for every $\alpha, \beta \in] a, b[, \alpha<\beta$.

Let $u:] a, b[\rightarrow \mathbb{R}$ be a continuous function and let there exist a finite or an infinite right, resp. left, limit of $u$ at the point $a$, resp. $b$. Then we will write $u(a+)$, resp. $u(b-)$, instead of $\lim _{t \rightarrow a+} u(t)$, resp. $\lim _{t \rightarrow b-} u(t)$.

$\widetilde{C}_{0}(] a, b[; \mathbb{R})$ is a set of all functions $u \in \widetilde{C}_{l o c}^{2}(] a, b[; \mathbb{R}) \cap C([a, b] ; \mathbb{R})$ such that there exist finite or infinite limits $u^{\prime}(a+)$ and $u^{\prime}(b-)$.

$L([a, b] ; \mathbb{R})$ is a Banach space of all Lebesgue integrable functions $p:[a, b] \rightarrow \mathbb{R}$ endowed with the norm

$$
\|p\|_{L}=\int_{a}^{b}|p(s)| \mathrm{d} s .
$$

$L\left([a, b] ; \mathbb{R}^{+}\right)=\left\{p \in L([a, b] ; \mathbb{R}): p(t) \in \mathbb{R}^{+}\right.$for a.e. $\left.t \in[a, b]\right\}$.

$\mathcal{L}_{a b}$ is a set of all linear bounded operators $\ell: C([a, b] ; \mathbb{R}) \rightarrow L([a, b] ; \mathbb{R})$.

$\mathcal{P}_{a b}$ is a set of all linear non-decreasing operators, i.e. operators $\ell \in \mathcal{L}_{a b}$ transforming the set $C\left([a, b] ; \mathbb{R}^{+}\right)$into the set $L\left([a, b] ; \mathbb{R}^{+}\right)$.

For any $x \in \mathbb{R}$, we put

$$
[x]_{+}=\frac{|x|+x}{2}, \quad[x]_{-}=\frac{|x|-x}{2} .
$$

An operator $\ell \in \mathcal{L}_{a b}$ is said to be a $t_{0}$-Volterra operator, where $t_{0} \in[a, b]$, if for every $a_{1} \in\left[a, t_{0}\right], b_{1} \in\left[t_{0}, b\right], a_{1} \neq b_{1}$ and $v \in C([a, b] ; \mathbb{R})$ satisfying

$$
v(t)=0 \quad \text { for } t \in\left[a_{1}, b_{1}\right]
$$

we have

$$
\ell(v)(t)=0 \quad \text { for a.e. } t \in\left[a_{1}, b_{1}\right] .
$$

Along with the problem (1.1), (1.2) we will consider the corresponding homogeneous problem

$$
\begin{aligned}
& u^{\prime \prime \prime}(t)=\ell(u)(t) \quad \text { for a.e. } t \in[a, b], \\
& u(a)=0, \quad u^{\prime}(a)=0, \quad u(b)=0 .
\end{aligned}
$$

From the general theory of boundary value problems, the following result is wellknown (see, e.g. $[1,3,7,8,10,16]$ ).

Theorem 1.1. The problem (1.1), (1.2) is uniquely solvable if and only if the corresponding homogeneous problem $\left(1.1_{0}\right),\left(1.2_{0}\right)$ has only the trivial solution. 
Definition 1.2. An operator $\ell \in \mathcal{L}_{a b}$ is said to belong to the set $\mathcal{V}([a, b])$ if every function $u \in \widetilde{C}^{2}([a, b] ; \mathbb{R})$ satisfying

$$
\begin{aligned}
& u^{\prime \prime \prime}(t) \leq \ell(u)(t) \quad \text { for a.e. } t \in[a, b], \\
& u(a) \geq 0, \quad u^{\prime}(a) \geq 0, \quad u(b) \geq 0
\end{aligned}
$$

admits the inequality

$$
u(t) \geq 0 \quad \text { for } t \in[a, b] .
$$

Definition 1.3. An operator $\ell \in \mathcal{L}_{a b}$ is said to belong to the set $\mathcal{V}_{0}([a, b])$ if every function $u \in \widetilde{C}^{2}([a, b] ; \mathbb{R})$ satisfying $(1.3)$ and

$$
u(a)=0, \quad u^{\prime}(a) \geq 0, \quad u(b)=0
$$

admits the inequality (1.5).

Remark 1.4. Obviously, $\mathcal{V}([a, b]) \subseteq \mathcal{V}_{0}([a, b])$. Moreover, if $-\ell \in \mathcal{P}_{a b}$ then

$$
\ell \in \mathcal{V}([a, b]) \quad \Longleftrightarrow \quad \ell \in \mathcal{V}_{0}([a, b]) \text {. }
$$

Indeed, let $-\ell \in \mathcal{P}_{a b}, \ell \in \mathcal{V}_{0}([a, b])$ and let $u \in \widetilde{C}^{2}([a, b] ; \mathbb{R})$ satisfy (1.3) and (1.4). Put

$$
\begin{aligned}
& v(t)=\frac{u(b)-u(a)}{(b-a)^{2}}(t-a)^{2}+u(a)+u^{\prime}(a) \frac{(b-t)(t-a)}{b-a} \quad \text { for } t \in[a, b], \\
& w(t)=u(t)-v(t) \quad \text { for } t \in[a, b] .
\end{aligned}
$$

Then

$$
\begin{aligned}
& v(t) \geq 0 \quad \text { for } t \in[a, b], \\
& w^{\prime \prime \prime}(t) \leq \ell(w)(t) \quad \text { for a.e. } t \in[a, b], \\
& w(a)=0, \quad w^{\prime}(a)=0, \quad w(b)=0 .
\end{aligned}
$$

Consequently, on account of (1.9), (1.10) and the assumption $\ell \in \mathcal{V}_{0}([a, b])$ we have $w(t) \geq 0$ for $t \in[a, b]$ which, in view of (1.7) and (1.8), implies (1.5).

Remark 1.5. If $\ell \in \mathcal{V}([a, b])$, resp. $\ell \in \mathcal{V}_{0}([a, b])$, then the problem (1.1), (1.2) is uniquely solvable. Indeed, let $u$ be an arbitrary solution to $\left(1.1_{0}\right),\left(1.2_{0}\right)$. Then, according to Definition 1.2, resp. Definition 1.3, the inequality (1.5) holds. However, $-u$ is also a solution to $\left(1.1_{0}\right),\left(1.2_{0}\right)$ and so

$$
-u(t) \geq 0 \quad \text { for } t \in[a, b] .
$$

Consequently, $u \equiv 0$ and the assertion follows from Theorem 1.1.

Notation 1.6. Let $\ell \in \mathcal{L}_{a b}$ be a $t_{0}$-Volterra operator, $\alpha \in\left[a, t_{0}\right], \beta \in\left[t_{0}, b\right]$, $\alpha \neq \beta$. Then we define a restriction $\ell^{\alpha \beta}$ of $\ell$ to the interval $[\alpha, \beta]$ by

$$
\ell^{\alpha \beta}(v)(t)=\ell(\vartheta(v))(t) \quad \text { for a.e. } t \in[\alpha, \beta], \quad v \in C([\alpha, \beta] ; \mathbb{R}),
$$

where

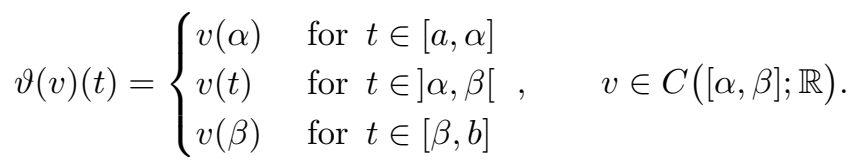


Obviously, $\ell^{\alpha \beta}: C([\alpha, \beta] ; \mathbb{R}) \rightarrow L([\alpha, \beta] ; \mathbb{R})$ is a linear bounded operator and $\ell^{\alpha \beta} \in \mathcal{P}_{\alpha \beta}$ whenever $\ell \in \mathcal{P}_{a b}$.

\section{Main Results}

Theorem 2.1. Let $-\ell \in \mathcal{P}_{a b}$. Then $\ell \in \mathcal{V}([a, b])$ if and only if there exists a function $\gamma \in \widetilde{C}_{0}(] a, b[; \mathbb{R})$ such that

$$
\begin{aligned}
& \gamma(t)>0 \quad \text { for } t \in] a, b[ \\
& \gamma^{\prime}(a+) \geq 0, \\
& \gamma^{\prime \prime \prime}(t) \leq \ell(\gamma)(t) \quad \text { for a.e. } t \in[a, b], \\
& \gamma(a)+\gamma^{\prime}(a+)+\gamma(b)+\text { meas }\left\{t \in[a, b]: \gamma^{\prime \prime \prime}(t)<\ell(\gamma)(t)\right\}>0 .
\end{aligned}
$$

Remark 2.2. According to Remark 1.4, the conditions of Theorem 2.1 are necessary and sufficient also for the inclusion $\ell \in \mathcal{V}_{0}([a, b])$ provided $-\ell \in \mathcal{P}_{a b}$.

Remark 2.3. Note that the zero operator, i.e. an operator defined by

$$
\ell(v)(t)=0 \quad \text { for a.e. } t \in[a, b], \quad v \in C([a, b] ; \mathbb{R}),
$$

belongs to the set $\mathcal{V}([a, b])$, because obviously, $-\ell \in \mathcal{P}_{a b}$ and $\gamma(t)=1$ for $t \in[a, b]$ satisfies (2.1)-(2.4).

Theorem 2.4. Let $\ell \in \mathcal{P}_{a b}$ be an a-Volterra operator. Then $\ell \in \mathcal{V}([a, b])$ if and only if there exists a function $\beta \in \widetilde{C}_{0}(] a, b[; \mathbb{R})$ such that

$$
\begin{aligned}
& \beta(t)>0 \quad \text { for } t \in[a, b[ \\
& \left.\beta^{\prime}(t) \leq 0 \quad \text { for } t \in\right] a, b[ \\
& \beta^{\prime \prime \prime}(t) \geq \ell(\beta)(t) \quad \text { for a.e. } t \in[a, b] .
\end{aligned}
$$

Theorem 2.5. Let $\ell \in \mathcal{P}_{a b}$ be an a-Volterra operator. Then $\ell \in \mathcal{V}_{0}([a, b])$ if and only if there exists a function $\beta \in \widetilde{C}_{0}(] a, b[; \mathbb{R})$ such that

$$
\begin{aligned}
& \beta(t)>0 \quad \text { for } t \in] a, b[, \\
& \beta^{\prime}(b-) \leq 0, \\
& \beta^{\prime \prime \prime}(t) \geq \ell(\beta)(t) \quad \text { for a.e. } t \in[a, b] .
\end{aligned}
$$

Conditions guaranteeing the inclusion

$$
\ell \in \mathcal{V}([a, b])
$$

resp.

$$
\ell \in \mathcal{V}_{0}([a, b])
$$

in the case when $\ell$ is a monotone operator, i.e. either $-\ell \in \mathcal{P}_{a b}$ or $\ell \in \mathcal{P}_{a b}$, are described in Theorems 2.1-2.5. Theorems 2.6 and 2.7 established below deal with the validity of the inclusion (2.11), resp. (2.12), in the case when $\ell$ can be expressed as a difference of two non-decreasing operators, i.e. in the case when

$$
\ell(v)(t)=\ell_{0}(v)(t)-\ell_{1}(v)(t) \quad \text { for a.e. } t \in[a, b], \quad v \in C([a, b] ; \mathbb{R}),
$$

with $\ell_{0}, \ell_{1} \in \mathcal{P}_{a b}$. 
Theorem 2.6. Let $\ell_{0}, \ell_{1} \in \mathcal{P}_{a b}$ and $\ell_{0},-\ell_{1} \in \mathcal{V}([a, b])$. Then the operator $\ell$ defined by $(2.13)$ belongs to the set $\mathcal{V}([a, b])$.

Theorem 2.7. Let $\ell_{0}, \ell_{1} \in \mathcal{P}_{a b}$ and $\ell_{0} \in \mathcal{V}_{0}([a, b]),-\ell_{1} \in \mathcal{V}([a, b])$. Then the operator $\ell$ defined by $(2.13)$ belongs to the set $\mathcal{V}_{0}([a, b])$.

According to Definitions 1.2 and 1.3 and Remark 1.5, the inclusion (2.11), resp. (2.12), guarantees that there exists a unique solution $u$ to the problem (1.1), (1.2). Moreover, if $q(t) \leq 0$ for a.e. $t \in[a, b]$ and $c_{i} \geq 0(i=1,2,3)$, resp. $c_{1}=0$, $c_{2} \geq 0, c_{3}=0$, then $u$ satisfies (1.5). More precisely, the following two assertions are valid.

Theorem 2.8. Let $\ell$ be defined by (2.13) with $\ell_{0}, \ell_{1} \in \mathcal{P}_{a b}$. Let, moreover,

$$
\ell_{0} \in \mathcal{V}([a, b]), \quad-\ell_{1} \in \mathcal{V}([a, b]) .
$$

Then the problem (1.1), (1.2) has a unique solution $u$. If, in addition,

$$
q(t) \leq 0 \quad \text { for a.e. } t \in[a, b]
$$

and

$$
c_{i} \geq 0 \quad(i=1,2,3)
$$

then (1.5) holds.

Theorem 2.9. Let $\ell$ be defined by (2.13) with $\ell_{0}, \ell_{1} \in \mathcal{P}_{a b}$. Let, moreover,

$$
\ell_{0} \in \mathcal{V}_{0}([a, b]), \quad-\ell_{1} \in \mathcal{V}([a, b]) .
$$

Then the problem (1.1), (1.2) has a unique solution $u$. If, in addition, (2.14) is fulfilled and

$$
c_{1}=0, \quad c_{2} \geq 0, \quad c_{3}=0,
$$

then (1.5) holds.

If we assume, in addition, that a non-decreasing part of the operator $\ell$ is $a$ Volterra, then $u$ is not only non-negative but even positive in $] a, b[$. More precisely, the following two assertions are valid.

Theorem 2.10. Let $\ell$ be defined by (2.13) with $\ell_{0}, \ell_{1} \in \mathcal{P}_{a b}$. Let, moreover, $\ell_{0}$ be an a-Volterra operator and

$$
\ell_{0} \in \mathcal{V}([a, b]), \quad-\ell_{1} \in \mathcal{V}([a, b]) .
$$

Then the problem (1.1), (1.2) has a unique solution $u$. If, in addition, the relations (2.14) and (2.15) are fulfilled with

$$
\|q\|_{L}+\sum_{i=1}^{3} c_{i}>0
$$

then

$$
u(t)>0 \quad \text { for } t \in] a, b[.
$$


Theorem 2.11. Let $\ell$ be defined by (2.13) with $\ell_{0}, \ell_{1} \in \mathcal{P}_{a b}$. Let, moreover, $\ell_{0}$ be an a-Volterra operator and

$$
\ell_{0} \in \mathcal{V}_{0}([a, b]), \quad-\ell_{1} \in \mathcal{V}([a, b]) .
$$

Then the problem (1.1), (1.2) has a unique solution $u$. If, in addition, the relations (2.14) and (2.16) are fulfilled with

$$
\|q\|_{L}+c_{2}>0
$$

then (2.18) holds.

The assumptions of Theorems 2.8 and 2.9 do not guarantee only the existence of a solution to (1.1), (1.2), but even the non-positivity of Green's operator of the problem (1.1), (1.20). If we are interested only in the solvability of the problem (1.1), (1.2), we can weaken the conditions of Theorems 2.8 and 2.9. More precisely, the following assertions are valid.

Theorem 2.12. Let $\ell$ be defined by (2.13) with $\ell_{0}, \ell_{1} \in \mathcal{P}_{a b}$. Let, moreover,

$$
\frac{1}{2} \ell_{0} \in \mathcal{V}_{0}([a, b]), \quad-\ell_{1} \in \mathcal{V}([a, b])
$$

Then the problem (1.1), (1.2) is uniquely solvable.

Theorem 2.13. Let $\ell$ be defined by (2.13) with $\ell_{0}, \ell_{1} \in \mathcal{P}_{a b}$. Let, moreover, $\ell_{0}$ and $\ell_{1}$ be $a$-Volterra operators and

$$
-\ell_{1} \in \mathcal{V}([a, b])
$$

Then the problem (1.1), (1.2) is uniquely solvable.

\section{Proofs}

To prove Theorem 2.1 we will need the following two lemmas.

Lemma 3.1. Let $w \in \widetilde{C}_{0}(] a, b[; \mathbb{R})$ be such that

$$
\begin{aligned}
& w \neq \equiv 0, \\
& w(t) \geq 0 \quad \text { for } t \in[a, b], \\
& w^{\prime \prime \prime}(t) \leq 0 \quad \text { for a.e. } t \in[a, b] .
\end{aligned}
$$

Then the following assertions are valid:

a) if

$$
w(a)=0, \quad w^{\prime}(a+)=0,
$$

then there exists a finite or an infinite limit

$$
w^{\prime \prime}(a+)>0
$$

b) if

$$
w^{\prime}(a+) \geq 0, \quad w(b)=0
$$

then

$$
w^{\prime}(b-)<0
$$


Proof. From (3.3) it follows that $w^{\prime \prime}$ is non-increasing. Therefore, there exist (finite or infinite) limits $w^{\prime \prime}(a+)$ and $w^{\prime \prime}(b-)$.

a) Assume that $w^{\prime \prime}(a+) \leq 0$. Then, according to (3.3) we have

$$
\left.w^{\prime \prime}(t) \leq 0 \quad \text { for } t \in\right] a, b[
$$

which together with (3.4) implies

$$
\left.w^{\prime}(t) \leq 0 \quad \text { for } t \in\right] a, b[
$$

and, consequently,

$$
w(t) \leq 0 \quad \text { for } t \in[a, b] .
$$

However, the last inequality together with (3.2) contradicts (3.1).

b) From (3.2) and (3.5) it follows that $w^{\prime}(b-) \leq 0$. Assume that $w^{\prime}(b-)=0$. Then, obviously, on account of $(3.2)$ we have $w^{\prime \prime}(b-) \geq 0$. Consequently, in view of (3.3), the inequality

$$
\left.w^{\prime \prime}(t) \geq 0 \quad \text { for } t \in\right] a, b[
$$

holds. However, the last inequality together with (3.5) implies

$$
\left.w^{\prime}(t) \geq 0 \quad \text { for } t \in\right] a, b[
$$

and, consequently,

$$
w(t) \leq 0 \quad \text { for } t \in[a, b] .
$$

The latter inequality and (3.2) result in $w \equiv 0$, which contradicts (3.1).

Lemma 3.2. Let $w \in \widetilde{C}_{0}(] a, b[; \mathbb{R})$ be such that (3.2) and (3.3) hold and let

$$
w^{\prime}(a+) \geq 0 .
$$

Let, moreover, there exist $\left.t_{0} \in\right] a, b[$ such that

$$
w\left(t_{0}\right)=0 .
$$

Then $w \equiv 0$.

Proof. Obviously, according to (3.2) and (3.7) we have

$$
w^{\prime}\left(t_{0}\right)=0, \quad w^{\prime \prime}\left(t_{0}\right) \geq 0 .
$$

Hence, in view of (3.3), we get

$$
\left.\left.w^{\prime \prime}(t) \geq 0 \quad \text { for } t \in\right] a, t_{0}\right]
$$

and, consequently, on account of (3.6) we obtain

$$
\left.\left.w^{\prime}(t) \geq 0 \quad \text { for } t \in\right] a, t_{0}\right] .
$$

The last inequality together with (3.7) implies

$$
w(t) \leq 0 \quad \text { for } t \in\left[a, t_{0}\right],
$$

whence, with respect to (3.2) we get

$$
w(t)=0 \quad \text { for } t \in\left[a, t_{0}\right] .
$$

Now (3.9) implies

$$
w^{\prime \prime}\left(t_{0}\right)=0
$$

which together with (3.3) yields

$$
w^{\prime \prime}(t) \leq 0 \quad \text { for } t \in\left[t_{0}, b[.\right.
$$


The last inequality with respect to (3.8) results in

$$
w^{\prime}(t) \leq 0 \quad \text { for } t \in\left[t_{0}, b[,\right.
$$

whence, in view of $(3.7)$, we obtain

$$
w(t) \leq 0 \quad \text { for } t \in\left[t_{0}, b\right] .
$$

Now (3.9) and (3.10), on account of (3.2), imply $w \equiv 0$.

Proof of Theorem 2.1. Let $\ell \in \mathcal{V}([a, b])$. Then according to Remark 1.5 there exists a unique solution $\gamma$ to the problem

$$
\begin{aligned}
& \gamma^{\prime \prime \prime}(t)=\ell(\gamma)(t) \quad \text { for a.e. } t \in[a, b], \\
& \gamma(a)=1, \quad \gamma^{\prime}(a)=0, \quad \gamma(b)=0 .
\end{aligned}
$$

Because $\ell \in \mathcal{V}([a, b])$, we have $\gamma(t) \geq 0$ for $t \in[a, b]$ and, moreover, according to Lemma 3.2 we find that $(2.1)$ holds. Thus $\gamma \in \widetilde{C}_{0}(] a, b[; \mathbb{R})$ and it satisfies (2.1)-(2.4).

Let there exist $\gamma \in \widetilde{C}_{0}(] a, b[; \mathbb{R})$ such that $(2.1)-(2.4)$ are fulfilled. Let, moreover, $u \in \widetilde{C}^{2}([a, b] ; \mathbb{R})$ be a function satisfying (1.3) and (1.4). We will show that (1.5) holds. Assume on the contrary that there exists $\left.t_{0} \in\right] a, b[$ such that

$$
u\left(t_{0}\right)<0 .
$$

Put

$$
\lambda=\sup \left\{-\frac{u(t)}{\gamma(t)}: t \in\right] a, b[\}
$$

Then, in view of (3.11), we have

$$
\lambda>0
$$

and according to (1.4), (2.1)-(2.3) and Lemma 3.1 we have $\lambda<+\infty$. Put

$$
w(t)=\lambda \gamma(t)+u(t) \quad \text { for } t \in[a, b] .
$$

Obviously,

$$
\begin{aligned}
& w(t) \geq 0 \quad \text { for } t \in[a, b], \\
& w^{\prime \prime \prime}(t) \leq \ell(w)(t) \quad \text { for a.e. } t \in[a, b] .
\end{aligned}
$$

We will show that

$$
\lambda>\limsup _{t \rightarrow a+} \frac{-u(t)}{\gamma(t)}
$$

Assume the contrary,

$$
\lambda=\limsup _{t \rightarrow a+} \frac{-u(t)}{\gamma(t)} .
$$

Then, in view of (1.4), (2.1), (2.2) and (3.12) we have

$$
u(a)=0, \quad u^{\prime}(a)=0, \quad \gamma(a)=0, \quad \gamma^{\prime}(a+)=0 .
$$

Now, according to (3.18) and Lemma 3.1 using l'Hospital rule we get

$$
\lambda=-\frac{u^{\prime \prime}(a)}{\gamma^{\prime \prime}(a+)} \text {. }
$$


Furthermore, (3.14)-(3.16), (3.18) and (3.19) imply

$$
\begin{array}{ll}
w^{\prime \prime \prime}(t) \leq 0 & \text { for a.e. } t \in[a, b], \\
w(a)=0, & w^{\prime}(a+)=0, \quad w^{\prime \prime}(a+)=0,
\end{array}
$$

which in view of $(3.15)$ results in

$$
w \equiv 0 .
$$

Moreover, (3.20) on account of (1.3), (1.4), (2.1), (2.3), (3.13) and (3.14) yields

$$
\begin{aligned}
& \gamma(b)=0, \\
& 0 \leq \ell(u)(t)-u^{\prime \prime \prime}(t)=\lambda\left(\gamma^{\prime \prime \prime}(t)-\ell(\gamma)(t)\right) \leq 0 \quad \text { for a.e. } t \in[a, b] .
\end{aligned}
$$

However, (3.18), (3.21) and (3.22) contradict (2.4).

Next we will show

$$
\lambda>\limsup _{t \rightarrow b-} \frac{-u(t)}{\gamma(t)} .
$$

Assume the contrary,

$$
\lambda=\limsup _{t \rightarrow b-} \frac{-u(t)}{\gamma(t)} .
$$

Then, in view of (1.4), (2.1) and (3.12) we have

$$
u(b)=0, \quad \gamma(b)=0 .
$$

Now, according to $(2.2),(3.24)$ and Lemma 3.1 using l'Hospital rule we get

$$
\lambda=-\frac{u^{\prime}(b)}{\gamma^{\prime}(b-)} .
$$

Furthermore, (3.14)-(3.16), (3.24) and (3.25) imply

$$
\begin{array}{ll}
w^{\prime \prime \prime}(t) \leq 0 & \text { for a.e. } t \in[a, b] \\
w(b)=0, & w^{\prime}(b-)=0 .
\end{array}
$$

Consequently, in view of (3.26) there exists a finite or an infinite limit $w^{\prime \prime}(b-)$. If $w^{\prime \prime}(b-)<0$, then there exists $\left.\varepsilon \in\right] 0, b-a[$ such that

$$
w^{\prime \prime}(t)<0 \quad \text { for } t \in[b-\varepsilon, b[,
$$

which together with (3.27) implies

$$
w(t)<0 \quad \text { for } t \in[b-\varepsilon, b[.
$$

However, (3.28) contradicts (3.15). Therefore, $w^{\prime \prime}(b-) \geq 0$ and so, on account of (3.26), we have

$$
\left.w^{\prime \prime}(t) \geq 0 \quad \text { for } t \in\right] a, b[.
$$

On the other hand, (1.4), (2.2) and (3.14) result in

$$
w^{\prime}(a+) \geq 0 .
$$

Now (3.27), (3.29) and (3.30) yield (3.20), which with respect to (1.3), (1.4), (2.1)-(2.3), (3.13) and (3.14) implies (3.18) and (3.22). However, (3.18), (3.22) and (3.24) contradict (2.4). 
In view of (3.17) and (3.23), from (3.12) and (3.14) it follows that there exists $\left.t_{0} \in\right] a, b\left[\right.$ such that $w\left(t_{0}\right)=0$. Consequently, according to Lemma 3.2 we have (3.20). However, as above (3.20) implies

$$
\begin{aligned}
& \gamma^{\prime \prime \prime}(t)=\ell(\gamma)(t) \quad \text { for a.e. } t \in[a, b], \\
& \gamma(a)=0, \quad \gamma^{\prime}(a+)=0, \quad \gamma(b)=0,
\end{aligned}
$$

which contradicts (2.4).

To prove Theorems 2.4 and 2.5 we will need the following two assertions.

Lemma 3.3. Let $w \in \widetilde{C}^{2}([a, b] ; \mathbb{R})$ be such that (3.1) and (3.2) hold. Let, moreover,

$$
\begin{aligned}
& w^{\prime \prime \prime}(t) \geq 0 \quad \text { for a.e. } t \in[a, b] \\
& w(b)=0 .
\end{aligned}
$$

Then

$$
w(t)>0 \quad \text { for } t \in] a, b[.
$$

Proof. Assume on the contrary, that there exists $\left.t_{0} \in\right] a, b[$ such that

$$
w\left(t_{0}\right)=0 .
$$

Then, in view of (3.2), we have

$$
\begin{aligned}
& w^{\prime}\left(t_{0}\right)=0, \\
& w^{\prime \prime}\left(t_{0}\right) \geq 0 .
\end{aligned}
$$

According to (3.31), from (3.36) it follows that

$$
w^{\prime \prime}(t) \geq 0 \quad \text { for } t \in\left[t_{0}, b\right],
$$

which together with (3.2), (3.32) and (3.34) implies

$$
w(t)=0 \quad \text { for } t \in\left[t_{0}, b\right]
$$

and, consequently,

$$
w^{\prime \prime}(t)=0 \quad \text { for } t \in\left[t_{0}, b\right] .
$$

Now (3.31), in view of $(3.38)$, results in

$$
w^{\prime \prime}(t) \leq 0 \quad \text { for } t \in\left[a, t_{0}\right] .
$$

On the other hand, on account of (3.2), (3.34), (3.35) and (3.39), we have

$$
0 \geq-w(a)=\int_{a}^{t_{0}} w^{\prime}(s) \mathrm{d} s=-\int_{a}^{t_{0}}(s-a) w^{\prime \prime}(s) \mathrm{d} s \geq 0
$$

and thus, in view of (3.39),

$$
w^{\prime \prime}(t)=0 \quad \text { for } t \in\left[a, t_{0}\right] .
$$

However, the last equality together with (3.34) and (3.35) implies

$$
w(t)=0 \quad \text { for } t \in\left[a, t_{0}\right]
$$

Now (3.37) and (3.40) contradict (3.1). 
Lemma 3.4. Let $w \in \widetilde{C}_{0}(] a, b[; \mathbb{R})$ be such that (3.31) and (3.33) hold. Let, moreover,

$$
\begin{aligned}
& w(a)=0, \\
& w^{\prime}(b-) \leq 0 .
\end{aligned}
$$

Then

$$
w^{\prime}(a+)>0 .
$$

Proof. Assume on the contrary that $w^{\prime}(a+) \leq 0$. In view of (3.33) and (3.41) we have

$$
w^{\prime}(a+)=0
$$

From (3.31) it follows that there exists a finite or an infinite limit $w^{\prime \prime}(a+)$. According to (3.33), (3.41) and (3.43) we have

$$
w^{\prime \prime}(a+) \geq 0 \text {. }
$$

Thus (3.31) and (3.44) yield

$$
\left.w^{\prime \prime}(t) \geq 0 \quad \text { for } t \in\right] a, b[,
$$

whence in view of (3.42) and (3.43) we get

$$
\left.w^{\prime}(t)=0 \quad \text { for } t \in\right] a, b[.
$$

However, the last equality together with (3.41) contradicts (3.33).

Proof of Theorem 2.4. Let $\ell \in \mathcal{V}([a, b])$. Then, according to Remark 1.5 there exists a unique solution $\beta$ to the problem

$$
\begin{aligned}
& \beta^{\prime \prime \prime}(t)=\ell(\beta)(t) \quad \text { for a.e. } t \in[a, b], \\
& \beta(a)=1, \quad \beta^{\prime}(a)=0, \quad \beta(b)=0 .
\end{aligned}
$$

Because $\ell \in \mathcal{V}([a, b])$, we have $\beta(t) \geq 0$ for $t \in[a, b]$ and, moreover, according to Lemma 3.3 we find that (2.5) holds. Furthermore, (2.5) and $\beta(b)=0$ imply $\beta^{\prime}(b) \leq 0$, which together with $\beta^{\prime}(a)=0$ and

$$
\beta^{\prime \prime \prime}(t) \geq 0 \quad \text { for a.e. } t \in[a, b]
$$

results in (2.6). Thus $\beta \in \widetilde{C}_{0}(] a, b[; \mathbb{R})$ and it satisfies $(2.5)-(2.7)$.

Let there exist $\beta \in \widetilde{C}_{0}(] a, b[; \mathbb{R})$ such that $(2.5)-(2.7)$ are fulfilled. Let, moreover, $u \in \widetilde{C}^{2}([a, b] ; \mathbb{R})$ be a function satisfying (1.3) and (1.4). We will show that (1.5) holds. Assume on the contrary that there exists $\left.t_{0} \in\right] a, b[\operatorname{such}$ that $(3.11)$ holds. According to (1.4) there exists $\left.\left.b_{0} \in\right] t_{0}, b\right]$ such that

$$
u\left(b_{0}\right)=0
$$

and

$$
u(t)<0 \quad \text { for } t \in\left[t_{0}, b_{0}[.\right.
$$

Moreover, in view of Notation 1.6, we have

$$
u^{\prime \prime \prime}(t) \leq \ell^{a b_{0}}(u)(t) \quad \text { for a.e. } t \in\left[a, b_{0}\right]
$$

and if $u(t) \leq 0$ for $t \in\left[a, b_{0}\right]$, then from (3.47) we get

$$
u^{\prime \prime \prime}(t) \leq 0 \quad \text { for a.e. } t \in\left[a, b_{0}\right]
$$


which together with (1.4) and (3.45), according to Remark 2.3, implies

$$
u(t) \geq 0 \quad \text { for } t \in\left[a, b_{0}\right] .
$$

However, (3.49) contradicts (3.46). Therefore, there exists $t_{1} \in\left[a, t_{0}[\right.$ such that

$$
u\left(t_{1}\right)>0 \text {. }
$$

Let $\left.b_{1} \in\right] t_{0}, b_{0}[$ be such that

$$
u\left(t_{0}\right)<u\left(b_{1}\right)
$$

and put

$$
\lambda=\sup \left\{\frac{u(t)}{\beta(t)}: t \in\left[a, b_{1}\right]\right\} .
$$

Obviously, according to (2.5) and (3.50) we have

$$
0<\lambda<+\infty \text {. }
$$

Furthermore,

$$
\lambda \beta(t)-u(t) \geq 0 \quad \text { for } t \in\left[a, b_{1}\right]
$$

and there exists $c \in\left[a, t_{0}[\right.$ such that

$$
\lambda \beta(c)-u(c)=0 .
$$

If $c \neq a$, then in view of (3.53) we have

$$
\lambda \beta^{\prime}(c+)-u^{\prime}(c)=0
$$

and

$$
\lambda \beta^{\prime \prime}(c+)-u^{\prime \prime}(c) \geq 0 .
$$

If $c=a$, then on account of (1.4), (2.6) and (3.52) we have

$$
\lambda \beta^{\prime}(a+)-u^{\prime}(a) \leq 0 .
$$

However, the last inequality together with (3.53) and (3.54) results in (3.55). Furthermore, in view of $(2.5),(2.7)$ and the assumption $\ell \in \mathcal{P}_{a b}$, there exists a finite or an infinite limit $\beta^{\prime \prime}(a+)$ and, consequently, (3.53)-(3.55) imply (3.56). Thus in both cases, (3.55) and (3.56) hold.

On the other hand, with respect to $(3.52)$,

$$
\lambda \beta^{\prime \prime \prime}(t)-u^{\prime \prime \prime}(t) \geq \ell^{a b_{1}}(\lambda \beta-u)(t) \quad \text { for a.e. } t \in\left[a, b_{1}\right],
$$

whence, in view of (3.53), we get

$$
\lambda \beta^{\prime \prime \prime}(t)-u^{\prime \prime \prime}(t) \geq 0 \quad \text { for a.e. } t \in\left[a, b_{1}\right] .
$$

Now (3.56) and (3.57) result in

$$
\left.\left.\lambda \beta^{\prime \prime}(t)-u^{\prime \prime}(t) \geq 0 \quad \text { for } t \in\right] c, b_{1}\right]
$$

and the latter inequality together with (3.55) yields

$$
\left.\left.\lambda \beta^{\prime}(t)-u^{\prime}(t) \geq 0 \quad \text { for } t \in\right] c, b_{1}\right] .
$$

From (3.58), according to (2.6) and (3.52), it follows that

$$
\left.\left.u^{\prime}(t) \leq 0 \quad \text { for } t \in\right] c, b_{1}\right]
$$

However, the last inequality contradicts (3.51). 
Proof of Theorem 2.5. Let $\ell \in \mathcal{V}_{0}([a, b])$. Then, according to Remark 1.5 there exists a unique solution $\beta$ to the problem

$$
\begin{aligned}
& \beta^{\prime \prime \prime}(t)=\ell(\beta)(t) \quad \text { for a.e. } t \in[a, b], \\
& \beta(a)=0, \quad \beta^{\prime}(a)=1, \quad \beta(b)=0 .
\end{aligned}
$$

Because $\ell \in \mathcal{V}_{0}([a, b])$, we have $\beta(t) \geq 0$ for $t \in[a, b]$ and, moreover, according to Lemma 3.3 we find that (2.8) holds. Furthermore, $(2.8)$ and $\beta(b)=0$ imply $(2.9)$. Thus $\beta \in \widetilde{C}_{0}(] a, b[; \mathbb{R})$ and it satisfies $(2.8)-(2.10)$.

Let there exist $\beta \in \widetilde{C}_{0}(] a, b[; \mathbb{R})$ such that $(2.8)-(2.10)$ are fulfilled. Let, moreover, $u \in \widetilde{C}^{2}([a, b] ; \mathbb{R})$ be a function satisfying (1.3) and (1.6). We will show that (1.5) holds. Assume on the contrary that there exists $\left.t_{0} \in\right] a, b[\operatorname{such}$ that $(3.11)$ holds. According to (1.6) there exists $\left.\left.b_{0} \in\right] t_{0}, b\right]$ such that (3.45) and (3.46) hold. Moreover, in view of Notation 1.6, we have (3.47) and if $u(t) \leq 0$ for $t \in\left[a, b_{0}\right]$, then from (3.47) we get (3.48) which together with (1.6) and (3.45), according to Remark 2.3, implies (3.49). However, (3.49) contradicts (3.46). Therefore, there exists $\left.t_{1} \in\right] a, t_{0}\left[\right.$ such that $(3.50)$ is fulfilled. Obviously, there exists $\left.b_{1} \in\right] t_{1}, b_{0}[$ such that

$$
\begin{aligned}
& u\left(b_{1}\right)<0, \\
& u^{\prime}\left(b_{1}\right)>0 \\
& u^{\prime \prime}\left(b_{1}\right) \geq 0 .
\end{aligned}
$$

Put

$$
\left.\left.\lambda=\sup \left\{\frac{u(t)}{\beta(t)}: t \in\right] a, b_{1}\right]\right\} .
$$

According to (2.8) and (3.50) we have

$$
\lambda>0 \text {. }
$$

Moreover, if $\beta(a)=0$, then according to Lemma 3.4 we have $\beta^{\prime}(a+)>0$ and, consequently, on account of (1.6), we find $\lambda<+\infty$. Put

$$
v(t)=\lambda \beta(t)-u(t) \quad \text { for } t \in\left[a, b_{1}\right]
$$

Then, obviously,

$$
v(t) \geq 0 \quad \text { for } t \in\left[a, b_{1}\right]
$$

and, moreover, with respect to (1.3), (2.10), (3.63), (3.64), and Notation 1.6, we have

$$
v^{\prime \prime \prime}(t) \geq \ell^{a b_{1}}(v)(t) \quad \text { for a.e. } t \in\left[a, b_{1}\right],
$$

whence in view of $(3.65)$ we get

$$
v^{\prime \prime \prime}(t) \geq 0 \quad \text { for a.e. } t \in\left[a, b_{1}\right]
$$

Furthermore, on account of (2.8), (3.59), (3.63) and (3.64), either there exists $c \in] a, b_{1}[$ such that

$$
v(c)=0,
$$

or

$$
\lambda=\limsup _{t \rightarrow a+} \frac{u(t)}{\beta(t)} .
$$


If (3.67) holds for some $c \in] a, b_{1}[$, then in view of (3.65) we have

$$
v^{\prime}(c)=0
$$

and

$$
v^{\prime \prime}(c) \geq 0 .
$$

If (3.68) holds, then in view of (1.6) and (3.63) we have

$$
\beta(a)=0
$$

and thus, according to Lemma 3.4,

$$
\beta^{\prime}(a+)>0 \text {. }
$$

Consequently, using l'Hospital rule, from (3.68) with respect to (1.6), (3.63), (3.71) and (3.72) we get

$$
\beta^{\prime}(a+)<+\infty, \quad \lambda=\frac{u^{\prime}(a)}{\beta^{\prime}(a+)} .
$$

Therefore, in view of (1.6), (3.64), (3.71) and (3.73) we have

$$
v(a)=0, \quad v^{\prime}(a+)=0 .
$$

According to (3.66) there exists a finite or an infinite limit $v^{\prime \prime}(a+)$. Assuming $v^{\prime \prime}(a+)<0$, on account of (3.74), we obtain a contradiction to (3.65). Therefore, $v^{\prime \prime}(a+) \geq 0$. Thus in both cases there exists $c \in\left[a, b_{1}[\right.$ such that

$$
v(c)=0, \quad v^{\prime}(c+)=0, \quad v^{\prime \prime}(c+) \geq 0 .
$$

Now (3.66) and (3.75) yield

$$
\left.\left.v^{\prime \prime}(t) \geq 0 \quad \text { for } t \in\right] c, b_{1}\right],
$$

whence, on account of (3.61), (3.63) and (3.64) we get

$$
\beta^{\prime \prime}\left(b_{1}\right) \geq 0 \text {. }
$$

Furthermore, (3.77) in view of (2.8) and (2.10) yields

$$
\beta^{\prime \prime}(t) \geq 0 \quad \text { for } t \in\left[b_{1}, b[,\right.
$$

whence with respect to $(2.9)$ we obtain

$$
\beta^{\prime}\left(b_{1}\right) \leq 0 .
$$

On the other hand, (3.76) together with (3.75) yields

$$
v^{\prime}\left(b_{1}\right) \geq 0
$$

whence in view of $(3.63),(3.64)$ and $(3.78)$ we get $u^{\prime}\left(b_{1}\right) \leq 0$ which contradicts (3.60).

Proof of Theorem 2.6. Let $u$ satisfy (1.3) and (1.4) with $\ell$ defined by (2.13). We will show that (1.5) holds. According to the assumption $\ell_{0} \in \mathcal{V}([a, b])$, in view of Remark 1.5, the problem

$$
\begin{aligned}
& \alpha^{\prime \prime \prime}(t)=\ell_{0}(\alpha)(t)+\ell_{1}\left([u]_{-}\right)(t) \quad \text { for a.e. } t \in[a, b], \\
& \alpha(a)=0, \quad \alpha^{\prime}(a)=0, \quad \alpha(b)=0
\end{aligned}
$$

has a unique solution $\alpha$ such that

$$
\alpha(t) \leq 0 \quad \text { for } t \in[a, b] .
$$


Put

$$
v(t)=\alpha(t)-u(t) \quad \text { for } t \in[a, b] .
$$

Then in view of (1.3), (1.4), (3.79), (3.80) and (3.82) we have

$$
\begin{aligned}
& v^{\prime \prime \prime}(t) \geq \ell_{0}(v)(t)+\ell_{1}\left([u]_{+}\right)(t) \quad \text { for a.e. } t \in[a, b], \\
& v(a) \leq 0, \quad v^{\prime}(a) \leq 0, \quad v(b) \leq 0 .
\end{aligned}
$$

Consequently, from (3.83) and (3.84), with respect to the inclusions $\ell_{0} \in \mathcal{V}([a, b])$ and $\ell_{1} \in \mathcal{P}_{a b}$, it follows that $v(t) \leq 0$ for $t \in[a, b]$, i.e. on account of $(3.82)$,

$$
\alpha(t) \leq u(t) \quad \text { for } t \in[a, b] .
$$

Now (3.81) and (3.85) result in

$$
\alpha(t) \leq-[u(t)]_{-} \quad \text { for } t \in[a, b] .
$$

Thus using (3.86) in (3.79) we get

$$
\alpha^{\prime \prime \prime}(t) \leq \ell_{0}(\alpha)(t)-\ell_{1}(\alpha)(t) \quad \text { for a.e. } t \in[a, b]
$$

and, with respect to the inclusion $\ell_{0} \in \mathcal{P}_{a b}$ and (3.81), the inequality (3.87) yields

$$
\alpha^{\prime \prime \prime}(t) \leq-\ell_{1}(\alpha)(t) \quad \text { for a.e. } t \in[a, b] .
$$

Now (3.88) together with (3.80) and the assumption $-\ell_{1} \in \mathcal{V}([a, b])$ implies

$$
\alpha(t) \geq 0 \quad \text { for } t \in[a, b],
$$

which together with $(3.85)$ results in (1.5).

Theorem 2.7 can be proven analogously to Theorem 2.6. Theorems 2.8 and 2.9 immediately follow from Theorems 2.6 and 2.7, Definitions 1.2 and 1.3, and Remark 1.5.

To prove Theorems 2.10 and 2.11 we will need the following assertions. The first one is a result from [17] and we formulate it in a suitable for us form.

Lemma 3.5. Let $\ell \in \mathcal{P}_{a b}$ be an a-Volterra operator. Then every function $w \in \widetilde{C}^{2}([a, b] ; \mathbb{R})$ satisfying

$$
\begin{aligned}
& w^{\prime \prime \prime}(t) \leq \ell(w)(t) \quad \text { for a.e. } t \in[a, b], \\
& w(a)=0, \quad w^{\prime}(a)=0, \quad w^{\prime \prime}(a)=0
\end{aligned}
$$

admits the inequality

$$
w(t) \leq 0 \quad \text { for } t \in[a, b] .
$$

Lemma 3.6. Let $\ell \in \mathcal{P}_{a b}$ be an a-Volterra operator, $\ell \in \mathcal{V}([a, b])$, and let $u \in \widetilde{C}^{2}([a, b] ; \mathbb{R})$ satisfy (1.3), (1.4). Let, moreover, there exist $\left.t_{0} \in\right] a, b[$ such that

$$
u\left(t_{0}\right)=0
$$

Then

$$
u(t)=0 \quad \text { for } t \in\left[t_{0}, b\right] .
$$


Proof. Put

Then, in view of (1.3),

$$
\widetilde{q}(t)=u^{\prime \prime \prime}(t)-\ell(u)(t) \quad \text { for a.e. } t \in[a, b] .
$$

$$
\widetilde{q}(t) \leq 0 \quad \text { for a.e. } t \in[a, b]
$$

and

$$
u^{\prime \prime \prime}(t)=\ell(u)(t)+\widetilde{q}(t) \quad \text { for a.e. } t \in[a, b] .
$$

According to Remark 1.5, there exists a unique solution $v$ to the problem

$$
\begin{aligned}
& v^{\prime \prime \prime}(t)=\ell(v)(t)+q_{0}(t) \quad \text { for a.e. } t \in[a, b], \\
& v(a)=0, \quad v^{\prime}(a)=0, \quad v(b)=u(b),
\end{aligned}
$$

where

$$
q_{0}(t)= \begin{cases}0 & \text { for a.e. } t \in\left[a, t_{0}[,\right. \\ \widetilde{q}(t) & \text { for a.e. } t \in\left[t_{0}, b\right] .\end{cases}
$$

Obviously, in view of (3.91) we have

$$
q_{0}(t) \leq 0 \quad \text { for a.e. } t \in[a, b],
$$

which, together with $(1.4),(3.93),(3.94)$ and the assumption $\ell \in \mathcal{V}([a, b])$, implies

$$
v(t) \geq 0 \quad \text { for } t \in[a, b] .
$$

On the other hand, put

$$
w(t)=u(t)-v(t) \quad \text { for } t \in[a, b] .
$$

Then, on account of (3.92)-(3.94) and (3.98), we have

$$
\begin{aligned}
& w^{\prime \prime \prime}(t)=\ell(w)(t)+\widetilde{q}(t)-q_{0}(t) \quad \text { for a.e. } t \in[a, b], \\
& w(a)=u(a), \quad w^{\prime}(a)=u^{\prime}(a), \quad w(b)=0 .
\end{aligned}
$$

Now (1.4), (3.91), (3.95), (3.99), (3.100) and the assumption $\ell \in \mathcal{V}([a, b])$ imply

$$
w(t) \geq 0 \quad \text { for } t \in[a, b] .
$$

However, (3.101) in view of (3.89), (3.97) and (3.98) yields

$$
v\left(t_{0}\right)=0 \text {. }
$$

Consequently, on account of (3.93)-(3.95), (3.97), (3.102) and the assumption $\ell \in \mathcal{P}_{a b}$, we have

$$
\begin{array}{ll}
v^{\prime \prime \prime}(t) \geq 0 & \text { for a.e. } t \in\left[a, t_{0}\right], \\
v(a)=0, & v^{\prime}(a)=0, \quad v\left(t_{0}\right)=0,
\end{array}
$$

whence, according to Remark 2.3, we get

$$
v(t) \leq 0 \quad \text { for } t \in\left[a, t_{0}\right] .
$$

Thus (3.97) and (3.103) result in

$$
v(t)=0 \quad \text { for } t \in\left[a, t_{0}\right] .
$$

Therefore,

$$
v^{\prime \prime}(a)=0 .
$$


Now from (3.93), (3.94), (3.96) and (3.104), according to Lemma 3.5, it follows that

$$
v(t) \leq 0 \quad \text { for } t \in[a, b] .
$$

However, (3.105) together with (3.97) implies

$$
v(t)=0 \quad \text { for } t \in[a, b] .
$$

Furthermore, (1.3), (1.4) and the assumption $\ell \in \mathcal{V}([a, b])$ result in (1.5) and thus from (3.89) we get

$$
u^{\prime}\left(t_{0}\right)=0 .
$$

Consequently, on account of (3.95), (3.98), (3.101) and the assumption $\ell \in \mathcal{P}_{a b}$, from $(3.89),(3.99),(3.100),(3.106)$ and (3.107) we obtain

$$
\begin{array}{ll}
w^{\prime \prime \prime}(t) \geq 0 & \text { for a.e. } t \in\left[t_{0}, b\right], \\
w\left(t_{0}\right)=0, & w^{\prime}\left(t_{0}\right)=0, \quad w(b)=0,
\end{array}
$$

whence, according to Remark 2.3, we get

$$
w(t) \leq 0 \quad \text { for } t \in\left[t_{0}, b\right] .
$$

However, (3.108) in view of (1.5), (3.98) and (3.106) results in (3.90).

The following assertion can be proved analogously.

Lemma 3.7. Let $\ell \in \mathcal{P}_{a b}$ be an a-Volterra operator, $\ell \in \mathcal{V}_{0}([a, b])$, and let $u \in \widetilde{C}^{2}([a, b] ; \mathbb{R})$ satisfy (1.3), (1.6). Let, moreover, there exist $\left.t_{0} \in\right] a, b[$ such that (3.89) is fulfilled. Then (3.90) holds.

Lemma 3.8. Let $\ell \in \mathcal{P}_{a b}$ and let $u \in \widetilde{C}^{2}([a, b] ; \mathbb{R})$ satisfy (1.3) and

$$
u(a)=0, \quad u(b)=0, \quad u^{\prime}(b)=0 .
$$

Let, moreover, there exist $\beta \in \widetilde{C}_{0}(] a, b[; \mathbb{R})$ satisfying

$$
\begin{aligned}
& \beta(t)>0 \quad \text { for } t \in] a, b], \\
& \beta^{\prime}(b-) \leq 0, \\
& \beta^{\prime \prime \prime}(t) \geq \ell(\beta)(t) \quad \text { for a.e. } t \in[a, b] .
\end{aligned}
$$

Then

$$
u(t) \leq 0 \quad \text { for } t \in[a, b] .
$$

Proof. Define the operators $\varphi: C([a, b] ; \mathbb{R}) \rightarrow C([a, b] ; \mathbb{R})$ and $\psi: L([a, b] ; \mathbb{R}) \rightarrow$ $L([a, b] ; \mathbb{R})$ by

$$
\begin{array}{ll}
\varphi(v)(t)=v(a+b-t) & \text { for } t \in[a, b], \\
\psi(v)(t)=v(a+b-t) & \text { for a.e. } t \in[a, b]
\end{array}
$$

and put

$$
\widetilde{\ell}(v)(t)=-\psi(\ell(\varphi(v)))(t) \quad \text { for a.e. } t \in[a, b], \quad v \in C([a, b] ; \mathbb{R}) .
$$

Furthermore, put

$$
\gamma(t)=\varphi(\beta)(t) \quad \text { for } t \in[a, b]
$$


Then, obviously, $\gamma \in \widetilde{C}_{0}(] a, b[; \mathbb{R})$ and on account of (3.110)-(3.112) we have

$$
\begin{aligned}
& \gamma(t)>0 \quad \text { for } t \in\left[a, b\left[, \quad \gamma^{\prime}(a+) \geq 0,\right.\right. \\
& \gamma^{\prime \prime \prime}(t) \leq \widetilde{\ell}(\gamma)(t) \quad \text { for a.e. } t \in[a, b] .
\end{aligned}
$$

Moreover, $-\tilde{\ell} \in \mathcal{P}_{a b}$. Consequently, according to Theorem 2.1, $\tilde{\ell} \in \mathcal{V}([a, b])$.

On the other hand, the function $w=\varphi(u)$, in view of (1.3) and (3.109) satisfies

$$
\begin{aligned}
& w^{\prime \prime \prime}(t) \geq \tilde{\ell}(w)(t) \quad \text { for a.e. } t \in[a, b], \\
& w(a)=0, \quad w^{\prime}(a)=0, \quad w(b)=0,
\end{aligned}
$$

whence, with respect to the inclusion $\tilde{\ell} \in \mathcal{V}([a, b])$, we obtain

$$
w(t) \leq 0 \quad \text { for } t \in[a, b] .
$$

However, the latter inequality yields (3.113).

Proof of Theorem 2.10. According to Theorem 2.8 we have that (1.1), (1.2) has a unique solution $u$ which satisfies (1.5) provided (2.14) and (2.15) hold. It remains to show that (2.17) implies (2.18).

Let $(2.14),(2.15)$ and $(2.17)$ hold and assume on the contrary that there exists $\left.t_{0} \in\right] a, b[$ such that (3.89) is satisfied. Because (1.5) and (2.13) are fulfilled, in view of $\ell_{1} \in \mathcal{P}_{a b},(2.14)$ and (2.15), from (1.1) and (1.2) we get (1.4) and

$$
u^{\prime \prime \prime}(t) \leq \ell_{0}(u)(t) \quad \text { for a.e. } t \in[a, b] .
$$

Thus, according to Lemma 3.6 we have (3.90).

On the other hand, according to the inclusion $\ell_{0} \in \mathcal{V}([a, b])$ and Remark 1.5 there exists a unique solution $\beta$ to the problem

$$
\begin{aligned}
& \beta^{\prime \prime \prime}(t)=\ell_{0}(\beta)(t) \quad \text { for a.e. } t \in[a, b], \\
& \beta(a)=1, \quad \beta^{\prime}(a)=0, \quad \beta(b)=0 .
\end{aligned}
$$

In the same way as in the proof of Theorem 2.4 one can show that $\beta$ satisfies (2.5) and (2.6).

First we will show that

Assume on the contrary that

$$
u(a)=0
$$

$$
u(a)>0
$$

Put

$$
w(t)=u(a) \beta(t)-u(t) \quad \text { for } t \in[a, b] .
$$

Then in view of (1.4), (3.90), (3.114)-(3.116), (3.118) and (3.119), we have

$$
\begin{aligned}
& w^{\prime \prime \prime}(t) \geq \ell_{0}(w)(t) \quad \text { for a.e. } t \in[a, b], \\
& w(a)=0, \quad w^{\prime}(a) \leq 0, \quad w(b)=0,
\end{aligned}
$$

whence on account of the inclusion $\ell_{0} \in \mathcal{V}([a, b])$ we obtain

$$
w(t) \leq 0 \quad \text { for } t \in[a, b] .
$$

However, the latter inequality with respect to (2.5), (3.118) and (3.119) results in

$$
u(t)>0 \quad \text { for } t \in] a, b[,
$$


which contradicts (3.90). Therefore (3.117) holds.

Now let $\left.b_{0} \in\right] t_{0}, b[$ be arbitrary but fixed. Then from (3.114) and (3.115), in view of (2.5), (2.6), (3.90) and (3.117), we get

$$
\begin{aligned}
& \beta^{\prime \prime \prime}(t)=\ell_{0}^{a b_{0}}(\beta)(t) \quad \text { for a.e. } t \in\left[a, b_{0}\right], \\
& u^{\prime \prime \prime}(t) \leq \ell_{0}^{a b_{0}}(u)(t) \quad \text { for a.e. } t \in\left[a, b_{0}\right], \\
& \left.\beta(t)>0 \quad \text { for } t \in] a, b_{0}\right], \\
& \beta^{\prime}\left(b_{0}\right) \leq 0, \\
& u(a)=0, \quad u\left(b_{0}\right)=0, \quad u^{\prime}\left(b_{0}\right)=0 .
\end{aligned}
$$

Consequently, according to Lemma 3.8,

$$
u(t) \leq 0 \quad \text { for } t \in\left[a, b_{0}\right]
$$

Now (1.5), (3.90) and (3.125) result in $u \equiv 0$, which contradicts (2.17).

Proof of Theorem 2.11. According to Theorem 2.9 we have that (1.1), (1.2) has a unique solution $u$ which satisfies (1.5) provided (2.14) and (2.16) hold. It remains to show that (2.19) implies (2.18).

Let $(2.14),(2.16)$ and $(2.19)$ hold and assume on the contrary that there exists $\left.t_{0} \in\right] a, b[$ such that (3.89) is satisfied. Because (1.5) and (2.13) are fulfilled, in view of $\ell_{1} \in \mathcal{P}_{a b},(2.14)$ and (2.16), from (1.1) and (1.2) we get (1.6) and (3.114). Thus, according to Lemma 3.7 we have (3.90).

On the other hand, according to the inclusion $\ell_{0} \in \mathcal{V}_{0}([a, b])$ and Remark 1.5 there exists a unique function $\beta \in \widetilde{C}^{2}([a, b] ; \mathbb{R})$ satisfying $(3.115)$ and

$$
\beta(a)=0, \quad \beta^{\prime}(a)=1, \quad \beta(b)=0 .
$$

In the same way as in the proof of Theorem 2.5 one can show that $\beta$ satisfies (2.8) and (2.9).

Now let $\left.b_{0} \in\right] t_{0}, b[$ be such that (3.123) holds. Such a point does exist because $\beta(b)=0$ and (2.8) holds. Then from (3.114) and (3.115), in view of (2.8), (3.90), (3.117) and (3.123), we get (3.120)-(3.124). Consequently, according to Lemma 3.8, (3.125) holds. Now (1.5), (3.90) and (3.125) result in $u \equiv 0$, which contradicts $(2.19)$.

The following two assertions are needed to prove Theorems 2.12 and 2.13.

Lemma 3.9. Let $-\ell \in \mathcal{P}_{a b}$ be an a-Volterra operator and let $\ell \in \mathcal{V}([a, b])$. Then for every $\left.b_{0} \in\right] a, b[$ the inclusion

$$
\ell^{a b_{0}} \in \mathcal{V}\left(\left[a, b_{0}\right]\right)
$$

holds.

Proof. According to Theorem 2.1 there exists $\gamma \in \widetilde{C}_{0}(] a, b[; \mathbb{R})$ satisfying $(2.1)$ (2.4). Let $\left.b_{0} \in\right] a, b[$ be arbitrary. Then from (2.1) we have

$$
\left.\gamma(t)>0 \quad \text { for } t \in] a, b_{0}\right]
$$

which also implies

$$
\gamma(a)+\gamma^{\prime}(a+)+\gamma\left(b_{0}\right)+\operatorname{meas}\left\{t \in\left[a, b_{0}\right]: \gamma^{\prime \prime \prime}(t)<\ell^{a b_{0}}(\gamma)(t)\right\}>0 .
$$


Because $\ell$ is an $a$-Volterra operator, from (2.3) we get

$$
\gamma^{\prime \prime \prime}(t) \leq \ell^{a b_{0}}(\gamma)(t) \quad \text { for a.e. } t \in\left[a, b_{0}\right] .
$$

Thus the restriction of $\gamma$ to the interval $\left[a, b_{0}\right]$ belongs to the set $\widetilde{C}_{0}(] a, b_{0}[; \mathbb{R})$ and it satisfies (2.2), (3.127)-(3.129). Therefore, according to Theorem 2.1, the inclusion (3.126) is valid.

The following assertion is a result from [10] and we formulate it in a suitable for us form.

Lemma 3.10. Let $\ell \in \mathcal{L}_{a b}$ be an a-Volterra operator for which there exists a function $\eta \in L\left([a, b] ; \mathbb{R}^{+}\right)$such that

$$
|\ell(v)(t)| \leq \eta(t)\|v\|_{C} \quad \text { for a.e. } t \in[a, b], \quad v \in C([a, b] ; \mathbb{R}) .
$$

Then the problem

$$
\begin{aligned}
& u^{\prime \prime \prime}(t)=\ell(u)(t) \quad \text { for } t \in[a, b], \\
& u(a)=0, \quad u^{\prime}(a)=0, \quad u^{\prime \prime}(a)=0
\end{aligned}
$$

has only the trivial solution.

Proof of Theorem 2.12. Let $u$ be a solution to the problem $\left(1.1_{0}\right),\left(1.2_{0}\right)$. According to Theorem 1.1 it is sufficient to show that $u \equiv 0$.

Because $\frac{1}{2} \ell_{0} \in \mathcal{V}_{0}([a, b])$, according to Remark 1.5 there exists a unique solution $\alpha$ to the problem

$$
\begin{aligned}
& \alpha^{\prime \prime \prime}(t)=\frac{1}{2} \ell_{0}(\alpha)(t)-\frac{1}{2} \ell_{0}(|u|)(t)-\ell_{1}(|u|)(t) \quad \text { for a.e. } t \in[a, b], \\
& \alpha(a)=0, \quad \alpha^{\prime}(a)=0, \quad \alpha(b)=0 .
\end{aligned}
$$

Put

$$
\begin{array}{ll}
w_{1}(t)=\alpha(t)-u(t) & \text { for } t \in[a, b], \\
w_{2}(t)=\alpha(t)+u(t) & \text { for } t \in[a, b] .
\end{array}
$$

Then from $\left(1.1_{0}\right),\left(1.2_{0}\right),(3.131),(3.132)$, in view of (3.133), (3.134) and the assumption $\ell_{0}, \ell_{1} \in \mathcal{P}_{a b}$, we get

$$
\begin{aligned}
& w_{1}^{\prime \prime \prime}(t) \leq \frac{1}{2} \ell_{0}\left(w_{1}\right)(t) \quad \text { for a.e. } t \in[a, b], \\
& w_{1}(a)=0, \quad w_{1}^{\prime}(a)=0, \quad w_{1}(b)=0, \\
& w_{2}^{\prime \prime \prime}(t) \leq \frac{1}{2} \ell_{0}\left(w_{2}\right)(t) \quad \text { for a.e. } t \in[a, b], \\
& w_{2}(a)=0, \quad w_{2}^{\prime}(a)=0, \quad w_{2}(b)=0 .
\end{aligned}
$$

Now from (3.135)-(3.138), on account of $\frac{1}{2} \ell_{0} \in \mathcal{V}_{0}([a, b])$, we get

$$
w_{1}(t) \geq 0 \quad \text { for } t \in[a, b], \quad w_{2}(t) \geq 0 \quad \text { for } t \in[a, b],
$$

whence in view of (3.133) and (3.134) we obtain

$$
\alpha(t) \geq|u(t)| \quad \text { for } t \in[a, b] .
$$

Now using (3.139) in (3.131) we find

$$
\alpha^{\prime \prime \prime}(t) \geq-\ell_{1}(\alpha)(t) \quad \text { for a.e. } t \in[a, b],
$$


which together with $(3.132)$ and the assumption $-\ell_{1} \in \mathcal{V}([a, b])$ implies

$$
\alpha(t) \leq 0 \quad \text { for } t \in[a, b] .
$$

Consequently, (3.139) and (3.140) yield $u \equiv 0$.

Proof of Theorem 2.13. Let $u$ be a solution to the problem $\left(1.1_{0}\right),\left(1.2_{0}\right)$. According to Theorem 1.1 it is sufficient to show that $u \equiv 0$.

Note that $\ell$ satisfies (3.130) with $\eta \equiv \ell_{0}(1)+\ell_{1}(1)$. Therefore, if $u^{\prime \prime}(a)=0$, then according to Lemma 3.10 we have $u \equiv 0$. Thus, without loss of generality we can assume that

$$
u^{\prime \prime}(a)>0 \text {. }
$$

According to $\left(1.2_{0}\right)$ and (3.141), there exists $\left.\left.b_{0} \in\right] a, b\right]$ such that

$$
\left.u\left(b_{0}\right)=0 \quad \text { and } \quad u(t)>0 \quad \text { for } t \in\right] a, b_{0}[\text {. }
$$

Therefore, in view of $\left(1.1_{0}\right),\left(1.2_{0}\right),(3.142)$ and because $\ell_{0}, \ell_{1} \in \mathcal{P}_{a b}$ are $a$-Volterra operators, we have

$$
\begin{aligned}
& u^{\prime \prime \prime}(t) \geq-\ell_{1}^{a b_{0}}(u)(t) \quad \text { for a.e. } t \in\left[a, b_{0}\right], \\
& u(a)=0, \quad u^{\prime}(a)=0, \quad u\left(b_{0}\right)=0 .
\end{aligned}
$$

However, according to Lemma 3.9, $-\ell_{1}^{a b_{0}} \in \mathcal{V}\left(\left[a, b_{0}\right]\right)$ and so (3.143) and (3.144) yield $u(t) \leq 0$ for $t \in\left[a, b_{0}\right]$, which contradicts (3.142).

\section{REFERENCES}

[1] N. V. Azbelev, V.P. Maksimov and L. F. Rakhmatullina, Introduction to the Theory of Functional Differential Equations (in Russian), Nauka, Moscow, 1991.

[2] N. V. Azbelev, V.P. Maksimov and L.F. Rakhmatullina, Methods of Modern Theory of Linear Functional Differential equations (in Russian), R\&C Dynamics, Moscow-Izhevsk, 2000 .

[3] E. Bravyi, A note on the Fredholm property of boundary value problems for linear functional differential equations, Mem. Differential Equations Math. Phys. 20 (2000), 133-135.

[4] E. I. Bravyi, Solvability of Boundary Value Problems for Linear Functional-differential Equations, R\&C Dynamics, Moscow-Izhevsk, 2011.

[5] Sh. Gelashvili and I. Kiguradze, On multi-point boundary value problems for systems of functional differential and difference equations, Mem. Differential Equations Math. Phys. 5 (1995), 1-113.

[6] R. Hakl, A. Lomtatidze and J. Šremr, Some Boundary Value Problems for First Order Scalar Functional Differential Equations, Folia Facul. Sci. Natur. Univ. Masar. Brun., Mathematica 10, Brno: Masaryk University, 2002.

[7] R. Hakl, A. Lomtatidze and I. P. Stavroulakis, On a boundary value problem for scalar linear functional differential equations, Abstract and Appl. Anal. 9 (2004), 45-67.

[8] R. Hakl and S. Mukhigulashvili, On a boundary value problem for $n$-th order linear functional differential systems, Georgian Math. J. 12 (2005), 229-236.

[9] J. Hale, Theory of Functional Differential Equations, Springer-Verlag, New YorkHeidelberg-Berlin, 1977.

[10] I. Kiguradze and B. Půža, On boundary value problems for systems of linear functional differential equations, Czechoslovak Math. J. 47 (1997), 341-373.

[11] I. Kiguradze and B. Půža, Boundary Value Problems for Systems of Linear Functional Differential Equations, Folia Facul. Sci. Natur. Univ. Masar. Brun., Mathematica 12, Brno: Masaryk University, 2003.

[12] V. Kolmanovskii and A. Myshkis, Applied theory of functional differential equations, Kluwer Academic Pub., Dordrecht-Boston-London, 1992. 
[13] V. Kolmanovskii and A. Myshkis, Introduction to the Theory and Applications of Functional Differential Equations, Kluwer Academic Pub., 1999.

[14] A. Lomtatidze and S. Mukhigulashvili, Some Two-point Boundary Value Problems for Second Order Functional Differential Equations, Folia Facul. Sci. Natur. Univ. Masar. Brun., Mathematica 8, Brno: Masaryk University, 2000.

[15] S. Mukhigulashvili, Two-point boundary value problems for second order functional differential equations, Mem. Differential Equations Math. Phys. 20 (2000), 1-112.

[16] Š. Schwabik, M. Tvrdý and O. Vejvoda, Differential and Integral Equations: Boundary Value Problems and Adjoints, Academia, Praha, 1979.

[17] J. Šremr, On systems of linear functional differential inequalities, Georgian Math. J. 13 (2006), 539-572.

[18] J. Šremr, On the initial value problem for two-dimensional linear functional differential systems, Mem. Differential Equations Math. Phys. 50 (2010), 1-127.

Robert Hakl, Institute of Mathematics, Academy of Sciences of the Czech Republic, Žižkova 22, 61662 Brno, Czech Republic

e-mail: hakl@ipm.cz 